\title{
COMPORTAMENTO DAS VAZÕES E TRANSPORTE DE SEDIMENTOS EM UM CANAL ABERTO ARTIFICIAL (PATROCÍNIO/MG) PARA DOIS EPISÓDIOS CHUVOSOS EM 2016
}

\author{
Renato Emanuel Silva ${ }^{(1)}$ Giliander Allan da Silva ${ }^{(2)}$, Jefferson Gomes Confesor ${ }^{(3)}$, Silvio Carlos \\ Rodrigues ${ }^{(4)}$
}

(1) Aluno Programa de Pós-graduação em Geografia/Universidade Federal de Uberlândia, renato.logan@gmail.com

(2) Aluno Programa de Pós-graduação em Geografia/Universidade Federal de Uberlândia, gili.franca@hotmail.com

(3) Aluno Programa de Pós-graduação em Geografia/Universidade Federal de Uberlândia, laggusa@ hotmail.com

(4) Professor Doutor do Instituto de Geografia/ Universidade Federal de Uberlândia, silgel@ufu.br

\section{EIXO: BACIAS HIDROGRÁFICAS E RECURSOS HÍDRICOS: ANÁLISE, PLANEJAMENTO E GESTÃO}

\section{Resumo}

\begin{abstract}
Pela abertura de canais artificiais em vertentes são alterados os escoamentos superficiais, difusos e/ou concentrados, o que resulta em influencias sobre os processos hidrogeomorfológicos responsáveis pela interface evolutiva da paisagem entre a água e as feições do relevo. É objetivo deste estudo, investigar como um canal aberto artificial, no município de Patrocínio (MG), se comporta para vazão e sedimentos ao longo de dois episódios pontuais de precipitação. Em busca de atender esse objetivo foram realizadas duas campanhas (uma com precipitação no período chuvoso e outra com precipitação isolada na estação seca) para obter dados de vazão e sedimentos suspensos no canal natural e na sua derivação artificial. Os resultados revelam que a existência de diques artificiais ao longo do canal artificial contribui para a desconexão desse com a vertente implicando em menores fluxos ao longo de sua calha.
\end{abstract}

Palavras chave: curso fluvial natural e artificial; hidrogeomorfologia; energia do canal; sedimento em suspensão

\section{Introdução}

Entre as ações humanas com potencial para alterar a dinâmica hidrogeomorfológica de determinados ambientes está a abertura de sulcos, como drenos, e de canais de transposição de água. Onde encontram-se tais elementos é necessário investigar os impactos que geram na bacia hidrográfica e como os mesmos ecoam nos comportamentos das vazões e sedimentos (CARVALHO, 2008).

Exemplificam essas interações, em ambientes de cerrado, pequenos açudes e canais empregados na derivação de águas para o atendimento rural. Estes canais são chamados de "regos d'água" e guardam relação com os canais de regadio portugueses. É interessante notar que essas estruturas raramente chamam 
a atenção de pesquisadores ligados a hidrogeomorfologia em bacias hidrográficas, portadoras destes elementos sem considera-los.

No município de Patrocínio-MG estes canais são comuns junto à bacia do rio Dourados (afluente do rio Paranaíba) sendo possível observar as principais características que os mesmos possuem quanto ao seu comportamento de transporte de água e sedimentos, bem como a ação humana na manutenção destes canais. Sendo objetivo deste estudo, investigar como um canal artificial, associado à dinâmica hidrogeomorfológica da vertente, se comporta quanto a vazão e sedimentos em episódios chuvosos. Tendo sido escolhido a derivação esquerda do córrego da Cava, afluente formador do rio Dourados em Patrocínio-MG. A figura 1 apresenta a localização da área de estudo e os pontos amostrais os quais permitiram o arranjo de análises utilizado neste trabalho.

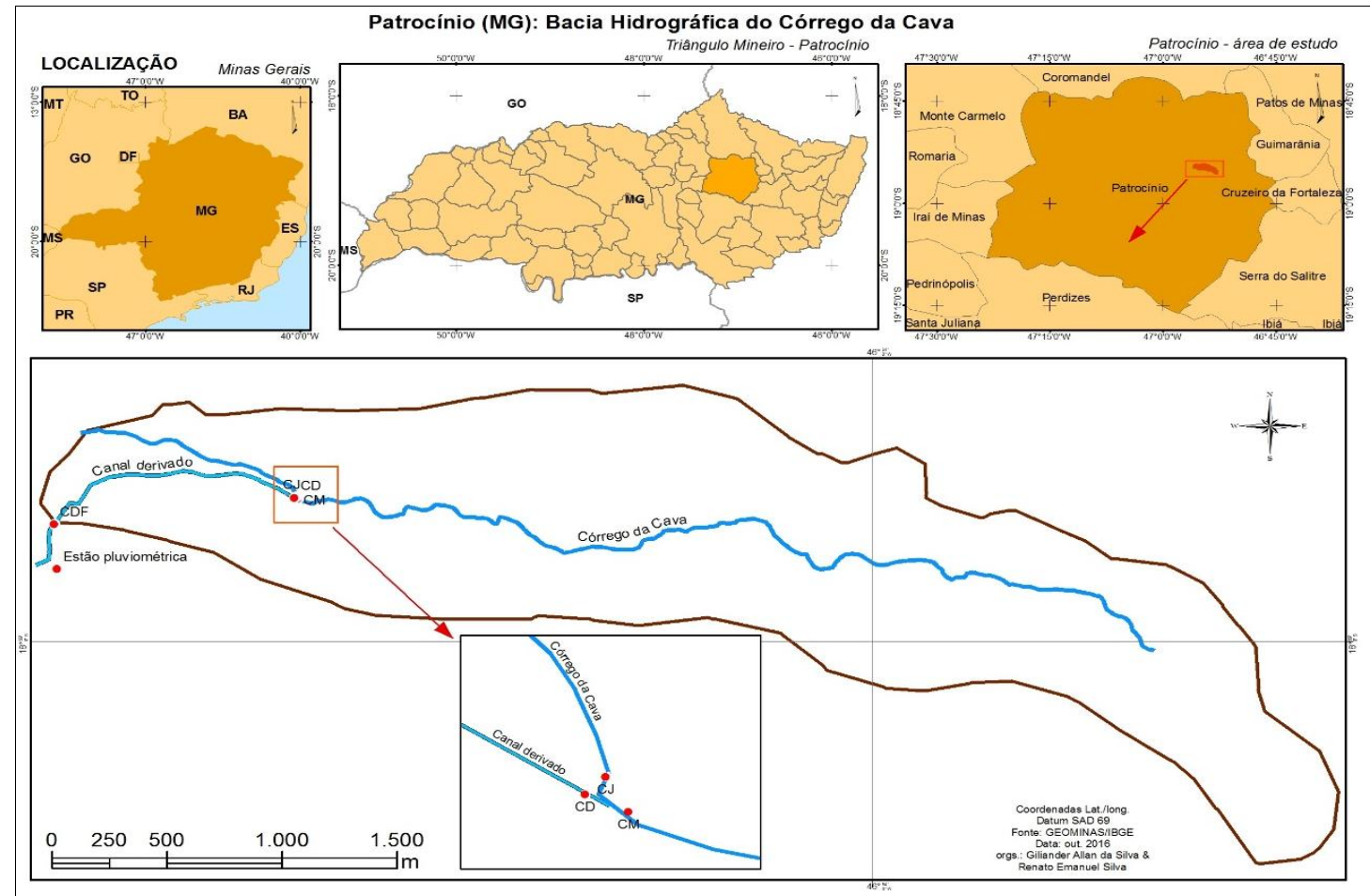

Figura 1: Localização da área de estudo. Fonte: próprio autor, 2016.

\section{Referencial teórico}

As assinaturas topográficas (Tarolli e Sofia, 2016), expressões de ações humanas, as quais incidem sobre a superfície e alteram as formas, promovendo mudanças na dinâmica da conectividade hidrológica (CROCKE e MOCKLER, 2001; MAAS e BROOKES, 2009; SIDLE e ONDA 2004). É o caso de estruturas como canais artificiais (tanto de transposição quanto de drenagem) que podem contribuir para o desenvolvimento de erosões. Nestes cenários as vertentes e os canais fluviais naturais conectados 


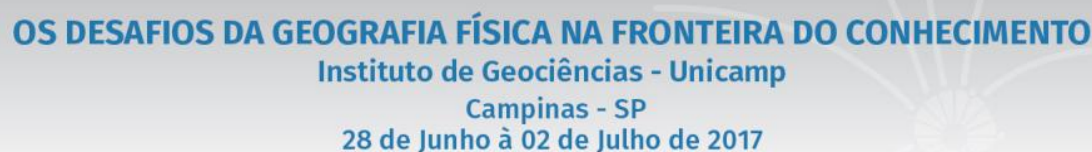

28 de Junho à 02 de Julho de 2017

de alguma forma aos canais artificiais sofrem influencias (CROCKE et al., 2005; THORP et al., 2009; POOLE, 2010).

Logo elementos como a vazão dos canais e o transporte de sedimentos podem sofrer significativas alterações em seu caminho rumo ao exutório das bacias alteradas. Tais parâmetros têm sido estudados como importante meio para conhecer o comportamento dos canais naturais permitindo investigar também a ação humana de alteração destes ambientes, os canais artificiais seriam interessantes e válidos exemplos destas mudanças como em outras alterações (CHOW, 1959; THORP et al., 2008; LENHART et al., 2010; MERTEN et al., 2014). Por isso importa investigar esses canais artificiais, para que sejam entendidos os impactos que produzem junto à bacia hidrográfica.

Contudo, o foco a estes canais tem sido voltado a questões relativas às temáticas sociais e bióticas. Como os estudos sobre a transposição do rio São Francisco, ou então, pesquisas voltadas à comunidade bentônica do canal do rio Piumhi (SOUZA e RIBEIRO 2004; FILHO e BUCKUP, 2005, NEVES e CARDOSO, 2009). Seria neste caso importante também investigar os impactos destes canais, em suas diferentes escalas e usos para temáticas relativas a sedimentos, vazões, como a hidrogeomorfologia é influencia por estes canais. São mais uma vez valorosas as sugestões de Tarolli e Sofia (2016), possibilitando entender como tais incisões impactam na dinâmica da vertente, pela alteração dos fluxos superficiais.

Assim, as vertentes podem influir no comportamento e na evolução dos canais no fundo de vale, os naturais. Exemplificam tal condição, Cunha e Thomaz (2009), que apresentaram como obras de contenção em estradas geram efeitos na turbidez e vazão de um canal fluvial. O’Hare et al. (2016) e Zong e Nepf (2010) avaliaram como a vegetação, presente pela vertente, pode implicar na dinâmica do fluxo ali existente e direcionado ao fundo de vale. Tendo sido estes exemplos comuns é valido agora considerar também a dinâmica dos canais abertos artificiais.

\section{Procedimentos metodológicos}

Os pontos amostrais foram escolhidos de modo a analisar o comportamento do canal natural antes (CM) e depois (CJ) do açude de derivação e no canal artificial imediatamente após o açude (CE) e em um ponto médio do mesmo (CEF). A seguir um esquema sugere os pontos de analise indicados de modo que se possam observar suas características (Figura 2).

Seguindo orientações de Lord et al. (2005) a pesquisa foi desenvolvida por meio de instrumentação provisória com acompanhamento de cenários específicos sem a construção de estruturas 


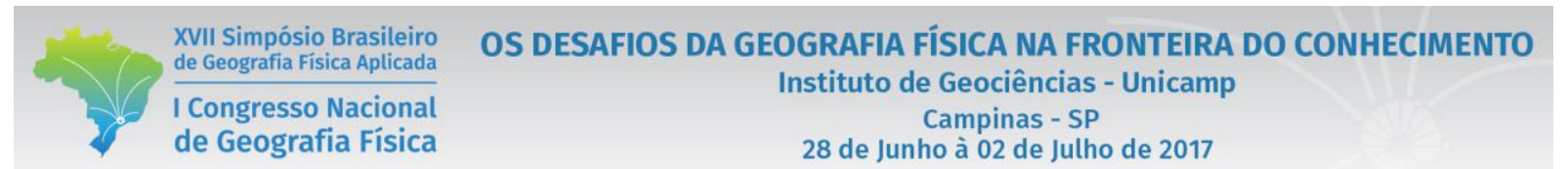

fixas o que facilita nos estudos de canais pequenos e com considerável comportamento mutável. As campanhas ocorreram em dois momentos chuvosos: um primeiro em 02/01/2016 e outro durante um cenário chuvoso em 03/06/2016 para coleta de dados de vazão, sedimentos em suspensão e observação do escoamento superficial nas vertentes.

Para as precipitações, o aferimento foi realizado em pluviômetro convencional, instalado nas proximidades do canal artificial pesquisado. Devido a estes trabalhos de campo, os responsáveis pela leitura dos índices pluviométricos, realizaram coletas de dados duas vezes ao dia, pela manhã e fim da tarde. Quanto aos sedimentos, foram coletadas três amostras de água em cada ponto amostral, seguindo metodologia, utilizando-se recipientes de um litro para a coleta do volume com amostragem pontual instantânea (Carvalho, 2008), posteriormente as amostras foram processadas no Laboratório de Geomorfologia e Erosão de Solos (LAGES/IG/UFU).
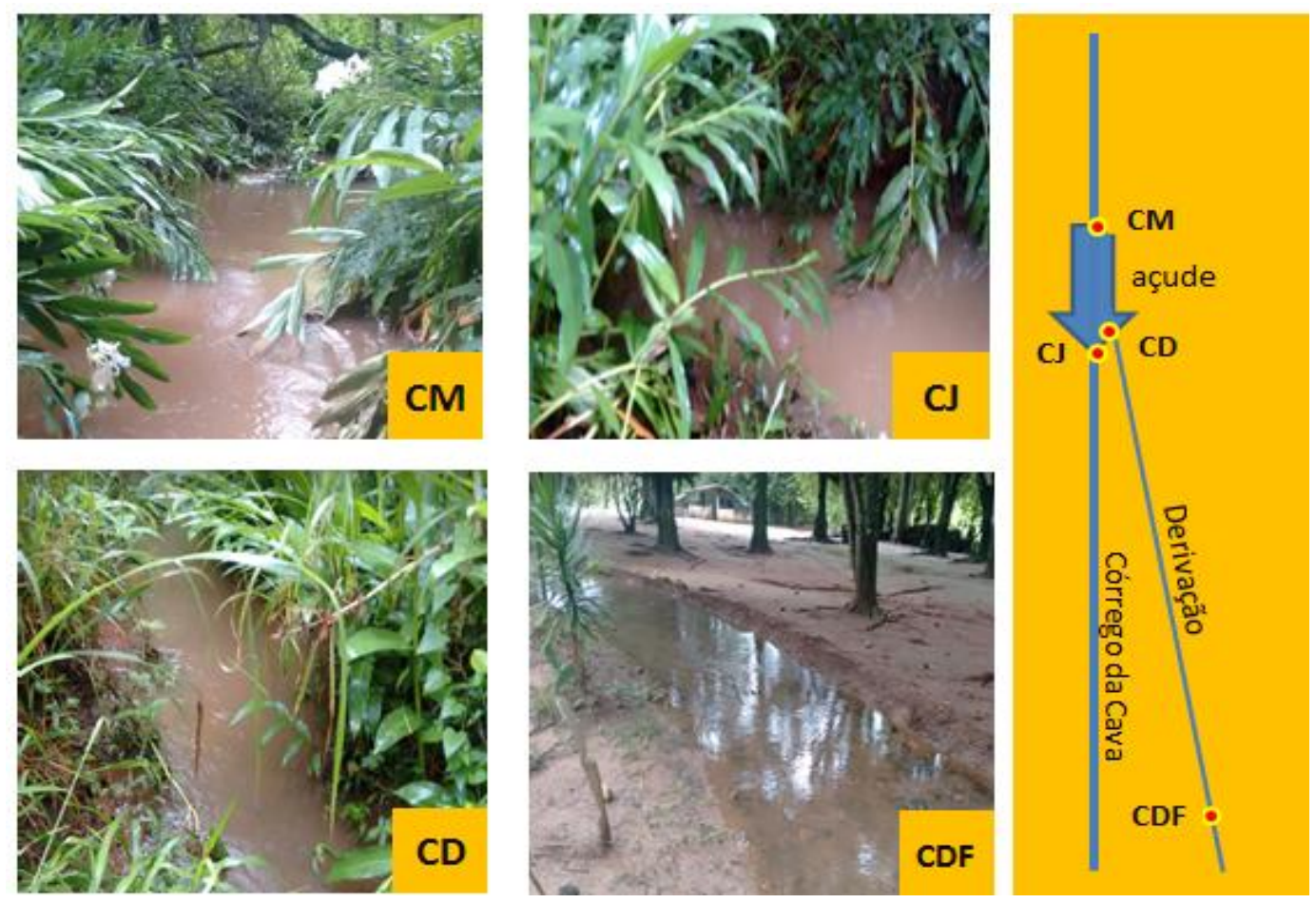

Figura 2: Locais para medição de vazões e amostragem. Fonte: próprio autor, 2016.

Para as vazões realizou-se medição a vau com relação da área da seção molhada e velocidade do fluxo, medida por meio do micromolinete fluviométrico (Global Water BC 1200), tendo sido definida, por batimetria, as seções molhadas na quais foram realizadas medições da velocidade do fluxo, os pontos amostrais variavam conforme a largura e profundidade da seção. 
Em laboratório, com temperatura de $25^{\circ} \mathrm{C}$, filtros de papel foram levados a estufa $\left(103^{\circ} \mathrm{C}\right)$ e posteriormente utilizados para filtragem das amostras de água coletadas. Ao termino do processo uma nova etapa de secagem na estuga obtendo se por diferença de pesos a quantidade de sedimentos encontrados nas amostras. Na fase de gabinete os dados de vazão, sedimentos e precipitação foram plotados a fim de permitir uma analise dos dois cenários.

\section{Resultados e Discussões}

A precipitação, nas 12 horas anteriores ao momento da medição do dia 02/01/2016, foi de $20 \mathrm{~mm}$, tendo sido registrado evento chuvosos ao longo das medições e coletas. Como se trata de um cenário específico foi necessário avaliar os dados de vazão e sedimentos, comparando os diferentes pontos do canal natural e artificial, de modo a observar como se comportavam tais questões. A tabela 1 apresenta os valores da vazão e dos sedimentos para os pontos amostrais no cenário de janeiro.

Tabela 1: cenários para vazão e precipitação de janeiro

\begin{tabular}{l|c|c|}
\hline cenário & $02 / 01 / 2016$ (estação chuvosa) \\
\hline preciptação & \multicolumn{2}{|c}{$20 \mathrm{~mm}$} \\
\hline \multicolumn{3}{|c|}{ variveis } \\
\hline Ponto & vazão ( $\mathrm{m} / \mathrm{s})$ & sed $(\mathrm{mg})$ \\
\hline $\mathrm{CM}$ & 0,096 & 0,036 \\
\hline $\mathrm{CJ}$ & 0,04 & 0,032 \\
\hline CE & 0,05 & 0,030 \\
\hline CEF & 0,0036 & 0,035 \\
\hline
\end{tabular}

A vazão nos permite observar que, no evento chuvoso em questão, os volumes de água foram mais direcionados para o canal derivado, isso ocorre pelo processo de manutenção do mesmo. Os moradores alargam as margens e aprofundam o leito de modo que um maior volume seja destinado naquela direção. Contudo é importante notar que mesmo em um período chuvoso com saturação do solo e escoamento superficial difuso e concentrado ocorrendo por diversos setores das vertentes a vazão reduziu para 7,2\% no ultimo ponto amostral em relação ao local de derivação.

Os motivos desta dinâmica são melhores respondidos considerando também os valores dos sedimentos suspensos. Nota-se que os valores de sedimentos (dados em mg), são reduzidos no açude de derivação, pois esta estrutura acaba por reduzir a velocidade do fluxo para menos de $0,2 \mathrm{~m} / \mathrm{s}$ o que contribui para a queda das partículas mais pesadas na coluna de água. Deste modo os valores de 


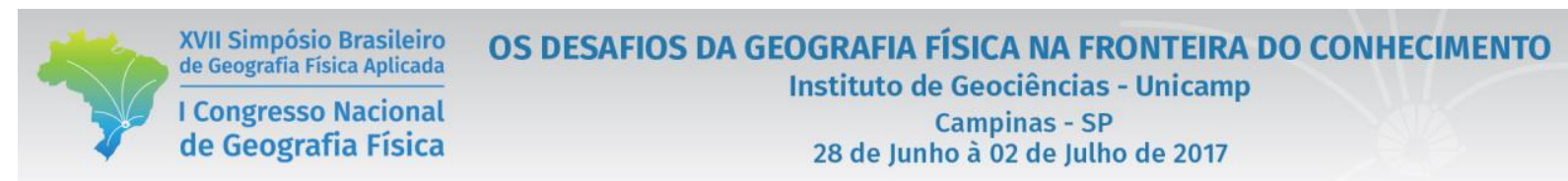

sedimentos em CJ e CE são menores que os localizados em CM. Contudo embora a vazão diminua em direção a CEF os valores ali para sedimentos são elevados.

Desta forma foi importante, na ocasião das coletas, ter percorrido o canal artificial para entender melhor sua dinâmica. Notou-se que a maior perda de água no canal era para vazamentos em seu ponto médio, a água por gravidade saia pelas aberturas, como danificações no canal artificial, e seguia de volta para o curso natural. Mas os valores elevados de sedimentos no trecho final da derivação deveriam sugerir então que os fluxos observados na vertente, ocasionados pelas precipitações, estavam acessando o sulco da derivação e elevando também a vazão.

Contudo, como revela a Figura 3 os canais contam, de sua metade em diante, com espécies de diques artificiais, resultado da limpeza periódica dos sedimentos. Dessa forma a enxurrada não consegue interagir diretamente com o canal artificial, tendo sido responsáveis pelos valores de sedimentos em CEF a presença de gado bovino, naquele setor, pisoteando o canal artificial.

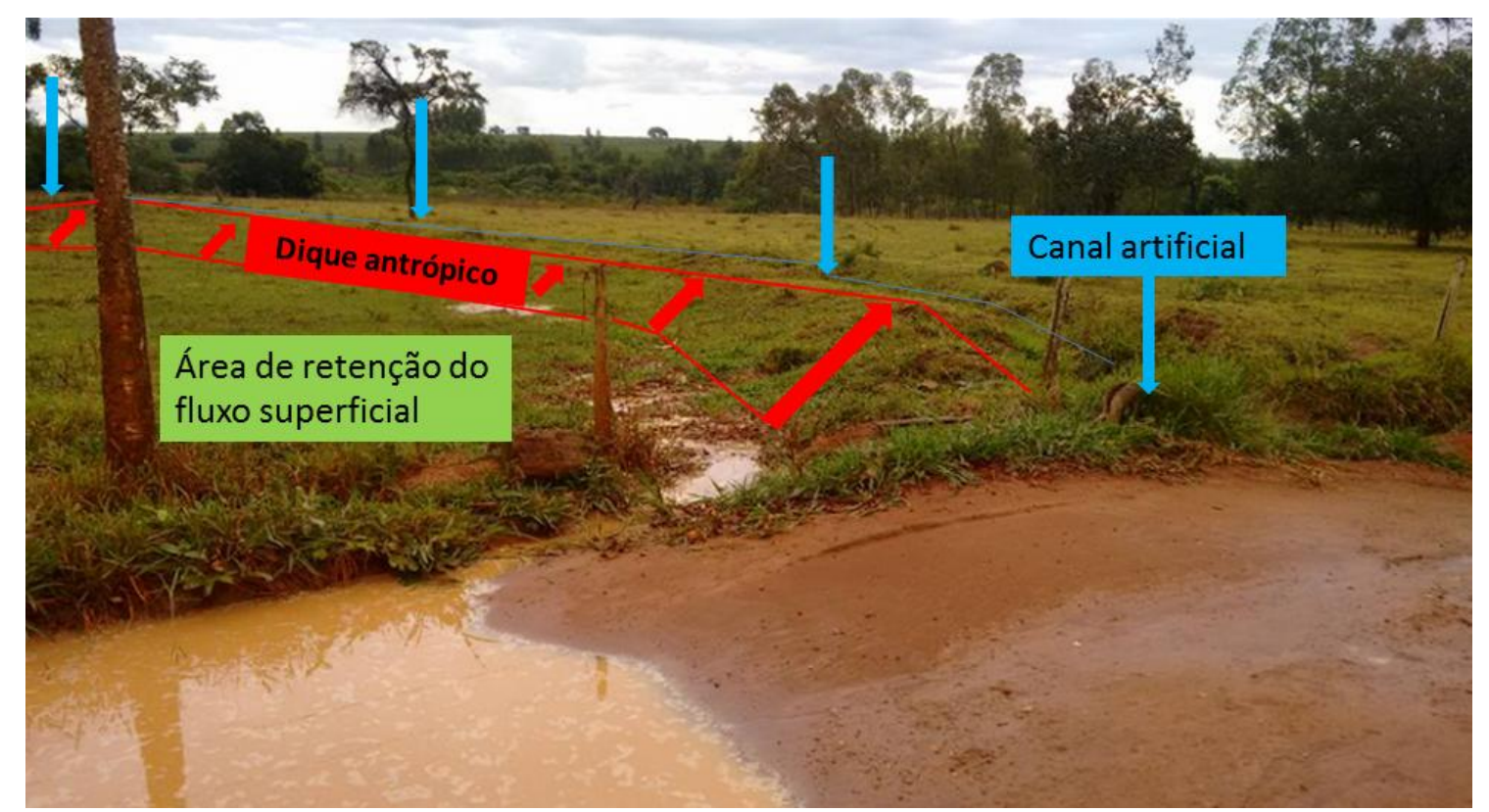

Figura 3: Em evento chuvoso é possível perceber que os diques artificias geram uma desconexão entre os fluxos das estradas e vertentes com o canal artificial.

Em junho foram realizadas as mesmas medições em um contexto diferente, agora a precipitação, $30 \mathrm{~mm}$ nas 12 horas antecedentes, não ocorria em um contexto chuvoso, mas no meio da estação seca. Desta forma foi possível observar algumas mudanças no comportamento do canal, mais uma vez influenciadas pela gestão dos moradores. 
Como sugere a tabela 2 os valores de vazão foram menores em CE, se comparados com CJ, resultado do crescimento da vegetação no canal artificial que diminuiu o fluxo de água em sua direção. Mas do ponto médio em diante o canal artificial foi limpo, tendo sido a vegetação e os sedimentos acomodados retirados. Deste modo o consumo e não os vazamentos foram responsáveis por uma redução menor se comparada à mesma configuração em janeiro.

Tabela 2: cenários para vazão e precipitação de junho

\begin{tabular}{l|c|c|}
\hline cenário & $03 / 06 / 2016$ (estação seca) \\
\hline preciptação & \multicolumn{2}{|c}{$30 \mathrm{~mm}$} \\
\hline \multicolumn{3}{|c}{ variveis } \\
\hline Ponto & vazão (m/s) & sed $(\mathrm{mg})$ \\
\hline CM & 0,32 & 0,032 \\
\hline CJ & 0,22 & 0,010 \\
\hline CE & 0,095 & 0,044 \\
\hline CEF & 0,022 & 0,045 \\
\hline
\end{tabular}

Para os sedimentos, notou-se valores elevados junto a $\mathrm{CE}$ e CEF, destoantes do contexto de $\mathrm{CM}$ e CJ, mais uma vez verificou-se o isolamento dos canais artificiais em relação as vertentes e presença de animais de grande porte gerando mudanças no comportamento destes canais. Nota-se, portanto, que caso não exista uma interferência direta no canal, pouco potencial de relação existe entre vertente e canal artificial dada aos diques formados em suas margens. Sendo o homem e as atividades a ele relacionadas as verdadeiras responsáveis por alterações na dinâmica hidrológica e sedimentar destas estruturas.

\section{Considerações finais}

Descobriu-se, por meio das análises realizadas, que os canais artificiais, pela existência de diques artificiais, pouco interagem com as vertentes sofrendo menores alterações de vazão e sedimentos, no contexto de eventos chuvosos. Ressalva-se que a presença de atividades dentro do canal (animais ou limpeza) podem mudar os índices dos sedimentos. Logo os canais funcionam como assinaturas topográficas capazes de interromper a circulação gravitacional da água em direção do fundo de vale. Alterando ainda, por meio de seus diques, a dinâmica hidrogeomorfológica também existente nos pequenos canais naturais. A presença destes canais deve ser portando considerada em estudos de escoamento superficial ou na temática da dinâmica da vazão de uma bacia hidrográfica, tendo em vista que a vazão não retorna concentrada aos canais naturais. 


\section{Agradecimentos}

Agradecem, os autores, a FAPEMIG por apoio financeiro para a participação neste evento. Os dois primeiros autores agradecem a CAPES por concessão de bolsa junto ao Programa de Pós-graduação do Instituto de Geografia (IG/UFU).

\section{Referências}

ARAUJO, I. B.; LIMA, A. M. M.; SANTOS, C. A. Caracterização hidrogeomorfológica das paisagens componentes da bacia hidrográfica do rio Tapajós. Enciclopédia biosfera, Centro Científico Conhecer Goiânia, v.11 n.22; p. 2015.

CARVALHO, N. O. Hidrossedimentologia Prática. Interciencia, Rio de Janeiro. 602 p. 2008.

CHOW, V. T. Open Channel Hydraulics McGraw-Hill. Book Company, Nova Iorque. 780 p, 1959.

CROCKE, J.; MOCKLER, S. Gully initiation and road-to-stream linkage in a forested catchment, southeastern Australia. Earth Surface Processes and Landforms, v. 26, p. 205-217, 2001.

CROCKE, J.; MOCKLER, S.; FOGARTY, P.; TAKKEN, I. Sediment concentration changes in runoff pathways from a forest road network and the resultant spatial pattern of catchment connectivity. Geomorphology, v. 68, n. 3-4, p. 257-268, 2005.

CUNHA, M.; THOMAZ, E. Análise da eficácia de caixas de contenção na redução de aporte de sedimento no rio das Pedras, Guarapuava-PR. Anais do $9^{\circ}$ Simposio Nacional de Geomorfologia, Rio de Janeiro, 2012. Disponível em: http://www.sinageo.org.br/2012/trabalhos/1/1-137-345.html. Acesso em: $09 / 02 / 2016$.

FILHO, O. M.; BUCKUP, P. A. A poorly known case of watershed transposition between The são Francisco and upper Paraná River basins. Neotropical ichthyology, 3(3) 449 - 452, 2005.

GURNELL, A.M.; CORENBLIT, D.; GARCÍA DE JALÓN, D.; GONZÁLEZ DEL TÁNAGO, M. ; GRABOWSKI, R.C. O'HARE, M.T.; SZEWCZYK, M. A conceptual model of vegetationhydrogeomorphology interactions within river corridors. River research and applications, 32: 142-163. 2016.

JANOCKO, M.; CARTIGNY, M.B.J.; NEMEC, W.; HANSEN, E.W.M. Turbidity current hydraulics and sediment deposition in erodible sinuous channels: Laboratory experiments and numerical simulations. Marine and Petroleum Geology, 222-249. 2013.

LENHART, C.F.; BROOKS, K.N.; HENELEY, D.; MAGNER, J.A. Spatial and temporal variation in suspended sediment, organic matter, and turbidity in a Minnesota prairie river: implications for TMDLs. Environ Monit Assess 165:435-447. 2010.

LORD, M. L.; DRU GERMANOSKI, D.; ALLMENDINGER, N. E. Fluvial geomorphology: Monitoring stream systems in response to a changing environment. Fluvial Geomorphology. The Geological Society of America, 36 p. 2009. 


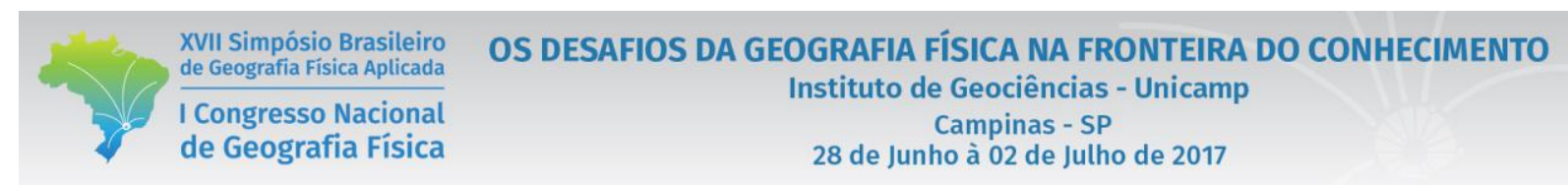

MAAS, S.; BROOKES, A. Fluvial geomorphology, FCERM Londres, 2009.

MAO, L.; CAVALLI, M.; COMITI, F. MARCHI, L. LENZI, M.A.; ARATTANO, M. Sediment transfer processes in two Alpine catchments of contrasting morphological settings. Journal of Hydrology, 364, 88-98, 2009.

MERTEN, G.H.; CAPEL. P.D. MINELLA, J.P.G. Effects of suspended sediment concentration and grain size on three optical turbidity sensors. J Soils Sediments, 14: 1235-1241. 2014.

MONTANHER, O. C.; SOUZA FILHO, E. E. Avaliação da alteração da concentração de sedimentos em suspensão em eventos de cheia do rio Paraná: o uso de imagens orbitais para avaliação de imapactos provocados pela barragem de Porto Primavera. Revista de Geografia. Recife: UFPE - DCG/NAPA, v. especial VIII SINAGEO, n. 2, Set. 2010.

NEVES, C.; CARDOSO, A. P. A experiência internacional com projetos de transposição de água - lições para a do rio São Francisco. XXIX Encontro Nacional de Egenharia de produção. Salvador. 2009.

O'HARE, M. T.; MOUNTFORD, J. O.; MAROTO, J.; GUNN, I. D. M. Plant traits relevant to fluvial geomorphology and hydrological interactions. River Res. Applic. 32: 179-189, 2016.

OLSON, P.L. City of wilkeson smp Update: channel migration assessment. Department of Ecology, Shorelands and Environmental assistance. 34 p. 2011.

POOLE, G. C. Stream hydrogeomorphology as a physical science basis for advances in stream ecology. J. N. Am. Benthol. Soc., v.29. 12-25. 2010.

SIDLE, R. C.; ONDA, Y. Hydrogeomorphology: overview of an emerging Science. Hydrological processes. 18, 597-602. 2004. Disponivel em: www.interscience.wiley.com. Acesso em: 09/02/2016.

SILVA, R. E. Disponibilidade e demanda hídrica a partir da análise ambiental da região do alto curso do rio Dourados em Patrocínio/MG. (Dissertação - Mestrado em Geografia). Universidade Federal de Uberlândia, Uberlândia. 2014.

SILVA, R. E.; RODRIGUES, S. C. Levantamento de estradas rurais e canais fluviais artificiais, em pequena bacia hidrográfica, e sua relação com o escoamento superficial. In: XXI Simpósio Brasileiro de Recursos Hídricos, 2015, Brasilia - DF. Trabalhos técnicos do XXI SBRH, 2015.

SOUZA, J. A.; RIBEIRO, E. Transposição do rio São Francisco e seus efeitos sobre o território. VII CBG, 2004.

TAROLLI, P.; SOFIA, G. Human topographic signatures and derived geomorphic processes across landscapes. Geomorphology, v. 255, p. 140-161, 2016.

THORP, J. H. The Riverine Ecosystem Synthesis Toward Conceptual Cohesiveness in River Science. Elsevier Inc. Amsterdam. 233 p. 2009.

ZONG, L.; NEPF, H. Flow and deposition in and around a finite patch of vegetation. Geomorphology, v. 116, 363-372, 2010. 